\title{
La guerre civile macédonienne de 1903-1908 et ses représentations dans l'historiographie nationale grecque
}

Tasos Kostopoulos

\section{(2) OpenEdition}

Journals

Édition électronique

URL : https://journals.openedition.org/ceb/835

DOI : $10.4000 /$ ceb.835

ISSN : 2261-4184

Éditeur

INALCO

\section{Édition imprimée}

Date de publication : 30 mars 2011

Pagination : 213-226

ISBN : 978-2-85831-189-7

ISSN : 0290-7402

\section{Référence électronique}

Tasos Kostopoulos, « La guerre civile macédonienne de 1903-1908 et ses représentations dans I'historiographie nationale grecque », Cahiers balkaniques [En ligne], 38-39 | 2011, mis en ligne le 06 décembre 2011, consulté le 06 juillet 2021. URL : http://journals.openedition.org/ceb/835 ; DOI : https://doi.org/10.4000/ceb.835

Ce document a été généré automatiquement le 6 juillet 2021

\section{(c) (i) (8)}

Cahiers balkaniques est mis à disposition selon les termes de la Licence Creative Commons Attribution - Pas d'Utilisation Commerciale 4.0 International. 


\title{
La guerre civile macédonienne de 1903-1908 et ses représentations dans l'historiographie nationale grecque
}

\author{
Tasos Kostopoulos
}

1 Dans les pays slaves des Balkans, le moment de l'histoire macédonienne moderne qui suscite le plus de commémorations est sans doute la révolte manquée d'Ilinden (en juillet 1903), revendiquée comme partie intégrante du patrimoine national de la Bulgarie et de la République de la Macédoine ex-yougoslave. En Grèce, pourtant, l'événement équivalent à commémorer n'est pas cette révolte contre le «joug ottoman ", mais, au contraire, la guerre civile entre communautés chrétiennes qui s'en est suivie.

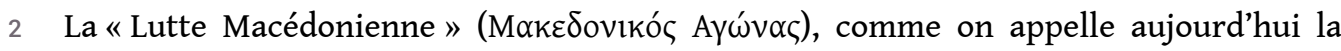
campagne déclenchée par les bandes de guérillas nationalistes entre 1904 et 1908 pour

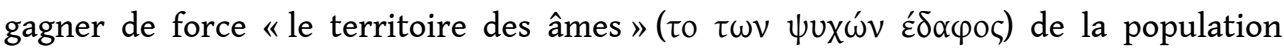
macédonienne ${ }^{1}$, a eu deux aspects bien différents, même s'ils étaient complémentaires.

3 Le premier aspect a été l'envoi par les gouvernements rivaux d'Athènes, de Sofia et de Belgrade des bandes organisées sur leur territoire pour affronter les groupes armés de l'adversaire, mais aussi pour exercer du terrorisme politique sur les populations pas seulement «ennemies", mais aussi «ambivalentes», dont la loyauté envers l'un ou l'autre camp national constituait la principale pomme de discorde. La transformation de cette rivalité, jusqu'alors plus ou moins pacifique, en une guerre ouverte de bandes armées a été déclenchée par l'intervention de l'Europe - et plus précisément, par l'article 3 du projet de réformes dit de Mürzsteg, imposé par les Puissances à la Porte Sublime en octobre 1903. Cet article prévoyait qu'« aussitôt qu'un apaisement du pays [la Macédoine] [serait] constaté ", les Puissances demanderaient "une modification dans la délimitation territoriale des unités administratives en vue d'un groupement plus régulier des différentes nationalités $»^{2}$. Cette disposition a été perçue par tous les intéressés comme le 
coup d'envoi du dépècement futur des irrédentistes macédoniens par les États chrétiens voisins.

4 Pour la Bulgarie, cette intervention a pris le caractère d'une politique d'entrisme dans les structures clandestines de l'Organisation révolutionnaire intérieure macédonienne (la fameuse ORIM), ayant comme but la récupération complète de l'appareil révolutionnaire qui avait organisé la révolte d'llinden et sa valorisation au profit du nationalisme bulgare. Ce processus d'entrisme datait des premières années d'existence de l'organisation, fondée en 1893, mais après Ilinden, il a pris une envergure décidément plus large, à cause de l'intensification des tendances «séparatistes ", provoquée par la passivité de la Bulgarie officielle pendant la révolte.

5 Pour la Grèce et la Serbie, au contraire, cette intervention a consisté surtout en l'envoi de bandes armées pour combattre l'influence bulgare sur les Slaves macédoniens et les activités révolutionnaires de l'ORIM.

6 Dans un premier temps de réflexion, les responsables grecs ont oscillé entre trois possibilités opposées :

7 Faire de l'entrisme dans l'ORIM, à l'instar de la Bulgarie, pour récupérer l'organisation au profit de la cause grecque, constituer des groupes armés locaux et grécophones pour participer à une seconde révolte de tous les Macédoniens, aux côtés des guérillas de l'ORIM, ou mener une campagne d'éradication des structures de l'ORIM au profit du statu quo.

8 Pendant cette période de réflexion, les Serbes sont allés jusqu'aux négociations avec l'ORIM et à une alliance éphémère avec la Bulgarie. Ensuite, les Andartès grecs et les Tchetniks serbes ont entrepris «l'œuvre de pacification" violente du pays et de " récupération » du "troupeau perdu » des anciens Patriarchistes qui étaient passés à l'Exarchat bulgare avant et - surtout - après la révolte d'Ilinden ${ }^{3}$.

9 Le second aspect du conflit concerne sa dimension en tant que guerre civile, à savoir le déchirement fratricide des communautés chrétiennes slavophones de la région convoitée. Cette dimension a été reconnue à l'époque par les principaux acteurs et par la plupart des observateurs extérieurs au conflit. Des cadres dirigeants de l'intervention grecque, comme Ion Dragoumis ou le consul à Monastir Dimitrios Kallergis, parlent par exemple dans leurs rapports d'une « guerre civile entre chrétiens » - projetée ou déjà commencée - en Macédoine ${ }^{4}$; la même formule est aussi utilisée par des écrivains comme John Foster Fraser ${ }^{5}$ ou par des hommes politiques des années suivantes, comme le chef du radicalisme libéral dans la Grèce de l'entre-deuxguerres, Alexandros Papanastasiou 6 .

10 Sur quels éléments est fondée cette idée de " guerre civile » ?

11 En premier, la confrontation armée entre partisans des nationalismes grec, bulgare et serbe s'est déroulée à l'intérieur d'un seul groupe ethnolinguistique de la Macédoine ottomane. Ce n'était pas un conflit interethnique, entre groupes ethnolinguistiques distincts, mais une bataille acharnée pour la suprématie politique - dite «nationale »-à l'intérieur d'un même groupe, celui des communautés slavophones de la région.

12 Un champ de bataille secondaire a été celui des communautés valaques ; la structure sociale très compacte de ce groupe et les liens étroits de protection ou couverture réciproques de ses membres ont pourtant réduit considérablement, dans ce cas-là, 
l'étendue des violences exercées. Les communautés grécophones, enfin, n'ont pas du tout été impliquées en tant que telles dans ce conflit qui ne les intéressait pas directement, dans la mesure où leur appartenance nationale dans la communauté imaginaire hellénique n'avait jamais fait l'objet d'une contestation. La participation des villageois macédoniens grécophones dans l'activité des bandes grecques a été ainsi plutôt limitée, provoquant un certain alarmisme chez les dirigeants de l'effort grec ${ }^{7}$. Encore plus limitée a été l'implication des Grecs dans les activités de l'ORIM, après l'ouverture officielle de l'organisation, en 1902, à « tous les éléments mécontents » de la région, «sans distinction de nationalité ${ }^{8}$; le phénomène des " comitadjis grecs » s'est restreint à quelques cas individuels, comme le propriétaire terrien de Meleniko, Manolis Kordopoulos, ou les réseaux de contrebandiers professionnels de la zone frontalière entre la Grèce et l'Empire ottoman'.

13 Un deuxième élément important: les sujets collectifs de cette confrontation armée entre Grecs, Bulgares et Serbes, à côté des bandes envoyées sur place par les États respectifs, sont des formations politiques rivales, constituées dans les communautés macédoniennes slavophones depuis les années 1860, que les sources de l'époque qualifient de "partis" nationaux: Parti bulgare, grec, serbe et - après $1904-$ macédonien ${ }^{10}$. Représentants de divers intérêts individuels ou collectifs, de stratégies sociales et d'orientations idéologiques divergentes au sein des communautés slavophones, ces partis nationaux se caractérisent par leur fonctionnement purement politique, la fluidité des choix individuels et la gradation ou l'inexistence d'engagement personnel de la part d'une grande partie de la population. Structurés autour d'un noyau d'adeptes motivés ou trop liés à des intérêts particuliers, ces partis mobilisent leurs troupes en mettant en avant des demandes sociales (pour ou contre le statu quo) et en développant des réseaux clientélistes classiques, où l'appartenance idéologique n'est qu'un prétexte qui cache la satisfaction des visées inavouées ${ }^{11}$.

14 Troisième élément important: comme dans toute guerre civile, on assiste au déchirement violent du tissu social avec des tueries ou des confrontations armées entre voisins, covillageois ou membres de la même famille. Les Mémoires des anciens combattants sont pleins de détails de ce genre : des Andartès grecs ayant des liens de parenté avec des comitadjis de l'ORIM; des tueries dues à des différends d'héritage entre frères, plutôt qu'à n'importe quelle conviction nationale; ou au contraire des cas de protection des paysans d'un des « partis nationaux » par leurs voisins ou parents du camp adverse, devant faire face aux groupes armés de leur propre camp; enfin, un deuil commun pour les victimes des deux camps qui pouvaient appartenir à une et même famille ${ }^{12}$.

Le traitement de cette dimension de " guerre civile » dans l'historiographie grecque est très éclairant sur la fonction idéologique que celle-ci (comme toute historiographie nationale) a été appelée à remplir au siècle dernier. Encore plus intéressantes sont les variations de ce traitement, les changements qu'il a subis dans le temps, en s'adaptant aux priorités et aux impératifs spécifiques de la politique nationale à chaque période. Il faut, donc, procéder à une périodisation de cette historiographie de la «lutte macédonienne » et l'examiner dans le contexte plus général de la politique dominante, en ce qui concerne à la fois la Macédoine et la Grèce dans son ensemble.

On doit, néanmoins, relever deux constantes observées pendant presque toute la période examinée ici. La première, c'est la domination totale et incontestable de la version historiographique du vainqueur (c'est-à-dire du nationalisme grec) et 
l'exclusion tout aussi totale des versions des vaincus, la dimension de guerre civile ayant attribué à cette répression idéologique et mnémonique un aspect d'urgence. La seconde constante est une perception de l'histoire, non pas comme reconstitution ou analyse du passé, mais comme profession de foi : on ne décrit pas ce qui c'est passé il y a cent ans, mais on exprime ses sentiments nationaux (et, peut-être, ses conceptions sociales) «sains » et "solides", en reproduisant la version des événements qui est considérée (ou projetée) comme " politiquement correcte » à un moment donné. Même après le rétablissement de la démocratie (plus exactement: l'instauration d'une démocratie véritable) dans les années 70 et son élargissement dans les années 80 , cette domination n'a pas été remise en question, car cela signifierait une prise de distance des communautés slavophones de la communauté imaginaire hellénique, soulevant à nouveau le spectre d'une marginalisation discriminatoire. La contradiction entre cette évolution et la rupture radicale qu'on constate après la restauration de la démocratie en ce qui concerne l'historiographie des années 40 est donc plus que frappante.

Revenons-nous à la périodisation du traitement de la "lutte macédonienne " par l'historiographie et la mémoire collective officielle grecques. Il faut distinguer quatre phases différentes, chacune correspondant à une période distincte de l'histoire du pays et de son évolution politique et intellectuelle.

La première phase est celle des années 1910 et de l'entre-deux-guerres. Elle commence par l'incorporation de la Macédoine du Sud dans le Royaume hellénique et se termine en 1941, par l'occupation de la Grèce par les forces de l'Axe (allemande, italienne et bulgare). Pendant cette période, la Macédoine grecque se transforme d'une province ottomane multilingue, multiconfessionnelle et multinationale, où l'élément grec et "grécisant » se trouve en minorité face aux "éléments étrangers ", en une province grecque typique, avec des minorités ethniques qui ne dépassent pas officiellement $11,2 \%$ de la population locale; transformation radicale, effectuée par des vagues successives d'épuration ethnique et - surtout - par l'échange réciproque, à grande échelle et obligatoire des minorités nationales entre la Grèce, la Turquie et la Bulgarie entre 1919 et $1926^{13}$. Après l'achèvement de l'échange, l'objectif le plus important que l'appareil étatique et les forces nationalistes de l'époque se fixent, est l'assimilation des minorités restées dans la région - des chrétiens slavophones surtout, évalués officiellement à 80.000 personnes et secrètement entre 160.000 et $200.000^{14}$. La campagne assimilatrice déclenchée prend alors deux formes : s'adonner à la répression et à l'assimilation linguistiques d'une part, visant à éradiquer le patois slave et imposer l'usage exclusif du grec; inculquer les conceptions fondamentales du nationalisme grec dans la conscience collective des «Grecs slavophones » de l'autre part ${ }^{15}$.

Tout traitement historiographique du conflit civil des années précédentes risquait, dans ces conditions, d'empêcher cette œuvre d'assimilation, en évoquant les clivages sanglants d'un passé plus ou moins récent. Étant donné que la moitié des slavophones restés en Grèce appartenaient, avant 1912, à l'Exarchat bulgare et avait participé à la "lutte macédonienne " dans le camp opposé, l'oubli officiel était perçu comme condition préalable, nécessaire à leur insertion (ou à celle de leurs enfants) dans l'imaginaire national grec ${ }^{16}$. Le traitement des victimes de la période d'avant 1912, dans les monuments érigés dans les villages, est caractéristique de la difficulté à incorporer la mémoire locale dans la mémoire officielle : à Zagoritchani (aujourd'hui Vassileiada) de Kastoria, par exemple, la liste des noms des morts pour la patrie inscrite sur le monument du village, érigé en 1931, comportait seulement les noms des soldats de 
l'armée régulière grecque, tombés après 1913 dans la Grande Guerre et la campagne de l'Asie Mineure ; les 10 morts du «Parti grec » avant 1912 ont été exclus, sans aucun doute pour ne pas faire penser aux 62 habitants du village qui avaient été massacrés par une bande grecque dans une tuerie aveugle en mars 1905. Un journal local, représentant l'opinion nationaliste de la région, a protesté en vain contre cette omission « inexplicable $»^{17}$.

Quelques facteurs complémentaires ont aussi contribué pendant l'entre-deux-guerres à l'exclusion de la « lutte macédonienne » de la mémoire officielle, à l'échelle nationale aussi bien que locale. Bien des détails de cette lutte continuaient par exemple à constituer des enjeux clairement politiques; la lutte de factions militaires entre Vénizelistes et Royalistes a ainsi conduit à des révisions successives de la liste officielle des anciens combattants en Macédoine, imposant des modifications importantes dans le récit officiel des actions armées du passé ${ }^{18}$. Le brigandage pratiqué par la plupart des bandes impliquées dans la lutte, ainsi que l'idée assez répandue que les cadres du Comité macédonien d'Athènes avaient détourné des fonds publics à leur profit semblent aussi avoir joué un rôle crucial dans la réticence des gouvernements de l'époque à mettre officiellement ses membres au panthéon national ${ }^{19}$. Et cela malgré la disponibilité des vétérans, regroupés autour d'une organisation fascisante (l'association «Pavlos Melas »), de participer à de nouvelles croisades contre l'ennemi intérieur juif et communiste. Bien entendu, leur offre n'a pas laissé indifférents les gouvernements des années trente ${ }^{20}$.

21 Le culte personnel du sous capitaine Pavlos Melas, le premier officier de l'armée grecque tombé en Macédoine - comme chef de bande - en octobre 1904 a été une exception à la règle. Fils et beau-fils de deux familles prépondérantes de l'aristocratie athénienne, membre de la société secrète Ethniki Etaireia, il est l'un des principaux responsables de la provocation armée qui a conduit à la guerre de 1897 contre la Turquie et à la défaite humiliante des armées helléniques. Pavlos Melas a été aussi un des principaux instigateurs de l'intervention armée de la Grèce en Macédoine après la révolte d'Ilinden. Il est vrai qu'il n'a pas eu le temps de faire grand-chose comme chef de bande, avant d'être tué dans des conditions très discutées pendant une escarmouche avec un détachement turc qui avait confondu sa bande avec les comitadjis de l'ORIM ; le fait qu'un membre illustre de la "bonne société " se soit sacrifié en Macédoine a néanmoins beaucoup contribué à la mobilisation nationaliste de l'époque ${ }^{21}$. En conséquence, Pavlos Melas a été transformé en héros national et symbole de la «lutte macédonienne ", bien avant l'incorporation de la Macédoine du Sud dans le Royaume de la Grèce. Pendant l'entre-deux-guerres, le culte de cette personne évidemment extraordinaire a ainsi servi de substitut à la commémoration de la «lutte macédonienne » en tant qu'événement collectif ${ }^{22}$.

22 La construction de la mémoire collective des événements, c'est donc la littérature qui l'a entreprise - à l'échelle locale par les nouvelles de Georgios Modis, et à l'échelle nationale par les romans (destinés aux enfants) de Pinelopi Delta. Modis, ancien combattant des bandes grecques, devenu homme politique influent du Parti libéral, sept fois élu député de Florina entre 1923 et 1956, a écrit 19 recueils de nouvelles dans lesquels il dépeint la lutte fratricide entre les partis grec et bulgare au début du siècle ; la dimension de guerre civile y est expressément accentuée, afin que le message de la "réconciliation» sous le régime bénéfique grec soit intégré par la population locale ${ }^{23}$. Pinelopi Delta, de son côté, préfère faire l'éloge des combattants venus de la Grèce libre 
ou de la diaspora grecque pour verser leur sang en Macédoine. Dans son principal roman, "Les secrets du marécage " (roman qui, avec ses 100 éditions et ses 500.000 exemplaires jusqu'à présent a formé plus que n'importe quelle autre source la conscience historique de la population grecque à propos de la question macédonienne), elle crée néanmoins le personnage emblématique du " petit Yovan ", enfant slavophone haïssant la nation bulgare, qui se révèle être l'enfant enlevé d'un combattant grec stratagème littéraire qui essaie d'expliquer le phénomène des "grécomans ", le noyau dur du "parti grec" dans les villages slavophones, d'une manière qui flatte le nationalisme grec ${ }^{24}$.

Si la dimension de guerre civile a été plus ou moins traitée par la littérature de l'entredeux-guerres, un autre aspect de la "lutte macédonienne » a été totalement censuré : l'alliance informelle entre les bandes grecques et le pouvoir ottoman, ravi de voir la guérilla révolutionnaire de l'ORIM et sa base sociale être décimées par des forces chrétiennes rivales. Dans les "secrets du marécage", Pinelopi Delta évite ainsi complètement de mentionner, même en passant, que la bataille finale de la lutte qu'elle décrit a été livrée en mai 1907 dans une opération commune de l'armée turque et des combattants grecs, opération militaire qui a eu lieu dans le temps narratif du récit.

La seconde phase commence avec l'occupation de la Grèce par les forces de l'Axe, en 1941, et se termine par la chute de la dictature militaire, en 1974. L'expérience de l'Occupation, de la résistance et de la guerre civile ont été déterminantes pour l'introduction de la « lutte macédonienne » au Panthéon national, comme le paradigme idéal qui éclairerait une histoire des combats successifs contre "la menace slave ». Cette «menace » a été présentée comme un danger constant et identique du temps de Byzance jusqu'à nos jours. Ce n'était plus alors la "lutte macédonienne" qui constituait une guerre civile, mais la guerre civile des années 40 qui était présentée comme une deuxième « lutte macédonienne » contre des barbares venus du Nord pour réduire les Grecs à l'esclavage slavo-communiste. Dans leurs mémoires ou dans leurs proclamations politiques, les anciens combattants des bandes grecques dans la Macédoine ottomane ne font d'habitude aucune distinction entre les comitadjis bulgaro-macédoniens du passé et les guérillas communistes du présent, même quand ces derniers se battent dans le Péloponnèse ou la Crète ${ }^{25}$. En plus (ce qui est resté complètement inconnu jusqu'à présent), l'appareil paramilitaire centralisé, constitué secrètement en juin 1946 pour organiser le massacre et les « disparitions » des cadres de gauche dans les villes et les villages du pays, et imposer le retour de la monarchie par le referendum truqué de la même année, a été baptisé par ses créateurs «Comité

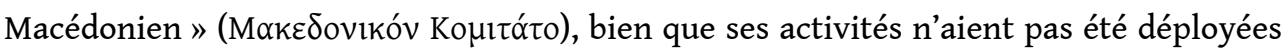
qu'en Macédoine, mais aussi en Grèce Centrale ${ }^{26}$.

Ce qu'on souligne, pendant cette période de guerre civile et de guerre froide, c'est surtout l'analogie entre la guérilla des «bandits» de l'ORIM et celle des "bandits communistes » des années 40 . Une seconde analogie lie les anciens comitadjis d'Ilinden qui ont changé de camp (en se transformant en Andartès grecs) avec les membres de la résistance de droite qui, pendant l'Occupation, sont aussi passés en 1943-44 de l'autre côté de la barrière, en coopérant plus ou moins ouvertement avec les autorités d'occupation. L'historiographie officielle grecque nie la dimension civile de l'ennemi, tout comme la politique officielle de l'après-guerre nie l'existence de toute minorité slave en Grèce du Nord : les mémoires des anciens combattants, qui sont publiées entre 1957 et 1963 par le très officiel Institut des Études balkaniques de Salonique (IMXA) 
sont ainsi sévèrement censurées par les éditeurs, pas seulement en ce qui concerne une bonne partie des atrocités grecques, mais aussi au niveau de la désignation des villages, dits «Bulgares $»^{27}$. En revanche, la production nationaliste locale était moins rigide, surtout quand des cadres locaux jugeaient nécessaire de comparer l'attitude patriotique progrecque de leurs ancêtres à celle, plus ambivalente, de leurs concurrents $^{28}$.

Il faut noter, enfin, que c'est pendant cette deuxième période que l'historiographie grecque de la « lutte macédonienne » est constituée comme telle, suivant un processus très centralisé (et placé sous le signe du "secret national ») qui n'aurait rien à envier aux méthodes utilisées pour la construction de l'histoire officielle dans les pays communistes voisins. La planification des futures éditions était organisée par une équipe des cadres du Ministère des Affaires étrangères, de l'Armée, des Services secrets et des Institutions de recherche officielles ou semi-officielles (comme IMXA ou la Société des Études macédoniennes ${ }^{29}$. Les versions finales de cette historiographie officielle ont circulé entre 1966 et 1979: en 1966, le livre de D. Dakin publié en anglais (par IMXA), en 1975, celui du général en retraite P. Tsamis (par la Société des Études macédoniennes) et en 1979, l'histoire officielle de « la lutte » par le Service historique de l'Armée ${ }^{30}$. Ces travaux, conçus pendant la guerre froide et la dictature militaire, représentent jusqu'à présent la version officielle des événements ${ }^{31}$.

La troisième période est celle de la Metapolitefsi, la transition démocratique des années 1974-1990. La chute de la junte a permis d'affranchir totalement la pensée politique et historique, en ôtant à la version officielle du nationalisme conservateur sa légitimité, sans remettre en question les fondements de l'idéologie nationale grecque. La « lutte macédonienne» a été complètement mise de côté par la nouvelle génération d'historiens, trop occupés à étudier les « trous noirs » des années 40 qu'on pouvait pour la première fois aborder sans la peur du gendarme ni celle des Cours de justice. La question (et surtout «la lutte») macédonienne reste, donc, un terrain de chasse réservé aux "experts» des instituts traditionnellement qualifiés, eux-mêmes marginalisés face aux nouveaux historiens, plutôt de gauche.

On est amené enfin à des analyses sérieuses du cadre international et balkanique de la question macédonienne, délivrées de la conception antérieure d'une confrontation gigantesque entre "hellénisme " et "panslavisme» à travers toute l'histoire de l'humanité ; la confrontation entre Grecs et Bulgares pour la Macédoine est désormais décrite comme une rivalité entre deux nationalismes qui essayaient de promouvoir leurs intérêts en arrachant le soutien des grandes puissances de l'époque.

En même temps, la dimension «intérieure » de la "lutte macédonienne " est passée sous silence. Le nouveau paradigme officiel, celui de la comparaison de la «lutte macédonienne » avec la révolution nationale de 1821, fondatrice de l'État-nation grec, a l'avantage de satisfaire le sentiment national sans créer de problèmes avec les voisins slaves qui, à partir de 1975, ont été considérés comme des alliés face à la Turquie ; ce même paradigme est pourtant incapable d'expliquer ce qui s'est passé en Macédoine ottomane au début du siècle, étant donné qu'il élimine totalement l'ennemi intérieur slave, en le remplaçant par le spectre des «bandes d'envahisseurs étrangers » sur un sol demeuré toujours hellénique.

30 En fait, après la chute de la dictature, on observe la consolidation de sa politique sur la question macédonienne, proclamée en 1969 à huit clos, et déclarant que toute référence à une personne (ou un objet) sous l'appellation de "macédonien» ne pourrait 
constituer qu'une version régionale du nom $\mathrm{Grec}^{32}$. La terminologie utilisée pour désigner la population locale - amie et ennemie - de 1904 est alors plutôt cryptique qu'explicative, privant le non-initié de toute compréhension du sujet. La doctrine nationale selon laquelle la question «dite macédonienne » est « inexistante » conduit donc à l'élimination complète de toute discussion historiographique réelle sur une facette du passé national, officiellement vénérée, mais en même temps érigée en tabou.

Une quatrième période est enfin peut-être née en 1992, avec l'éclatement de la nouvelle question macédonienne sur la reconnaissance officielle et le nom de la République exyougoslave de Macédoine. Cette évolution politique a revigoré l'intérêt scientifique sur la question macédonienne et engendré une génération de nouveaux venus sur le terrain qui ne se sentent pas du tout obligés de respecter les clichés et autres stéréotypes existants, formulés pendant la guerre civile ou la guerre froide ${ }^{33}$. Ce courant « révisionniste ", qui essaie de reconstituer le paysage social et les enjeux réels de la Macédoine des débuts du vingtième siècle, n'est pourtant pas encore dominant au niveau académique, sans parler de la société en général. Malheureusement, le dialogue avec les historiographies bulgare et macédonienne (de la République ex-yougoslave de Macédoine) a été aussi impossible, car ces dernières - à la différence d'une partie de la nouvelle historiographie turque, par exemple - ne semblent pas du tout jusqu'à présent disposées à remettre en question les stéréotypes nationalistes et les mythes nationaux de leur propre patrie.

En Grèce, les gardiens du statu quo historiographique ont cependant considéré l'émergence de ce nouveau courant comme une provocation et ont déclenché, il y a trois ans, une "contre-attaque " médiatique à échelle nationale, avec comme objectif déclaré de "délivrer» le système éducatif des "traîtres» qui subvertissent la mythologie nationale. Une mythologie nationale déclarée absolument nécessaire pour combattre les «forces de la mondialisation" (perçues comme intrinsèquement contraires aux intérêts nationaux grecs) et autres dangers.

Mais cette dernière évolution est une affaire que l'on n'a pas le temps d'aborder ici.

\section{NOTES}

1. Pour « le territoire des âmes » comme enjeu du conflit : IAYE 1904/AAK/ $\Sigma \tau^{\prime}$, L. Koromilas au MAE, Salonique 30.9.1904, $\mathrm{n}^{\circ} 11$ spécial; cf. aussi Dimitris Livanios, "Conquering the souls: Nationalism and Greek guerilla warfare in Ottoman Macedonia, 1904-1908”, Byzantine and Modern Greek Studies, 23 (1999), pp. 195-221.

2. Pour l'article 3, voir Atanas Schopoff, Les réformes et la protection des Chrétiens en Turquie, 1673-1904, Paris, 1904, p. 575. La perception générale était que cette formulation constituait un coup d'envoi pour le dépeçage prochain de la Macédoine ottomane ; voir IAYE 1904/52, Kypraios

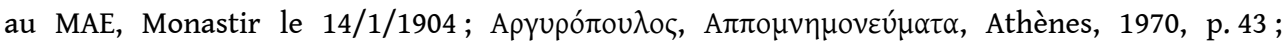

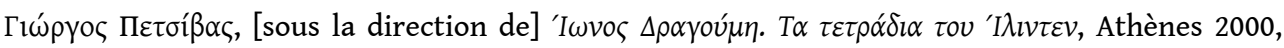
p.622; ОСВОБОДИТЕЛНАТА БОРБА НА БЪЛГАРИТЕ В МАКЕДОНИЯ И ОДРИНСКО, 1902-1904. ДИПЛОМАТИЧЕСКИ ДОКУМЕНТИ, Sofia, 1978, pp. 538-40 ; ПАНЧО ДОРЕВ, КОСТУРСКО В МАКЕДОНСКАТА 
РЕВОЛЮЦИЯТА, Sofia 1937, p. 57 ; СИМОН ДРАКУЛ, МАКЕДОНИјА МЕЃУ АВТОНОМИјАТА И ДЕЛЕЖОТ, vol. III (1904), Kumanovo 1997, p. 113.

3. La bibliographie sur le sujet est vraiment immense. Parmi les œuvres principales de référence : Fikret Adanır, Die Makedonische Frage, Wiesbaden, 1979; Duncan Perry, The politics of terror. The Macedonian revolutionary movement, 1893-1903, Londres, 1988 ; Douglas Dakin, The Greek Struggle in

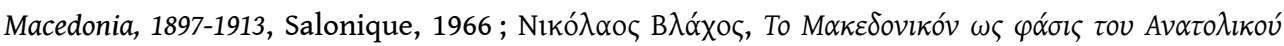

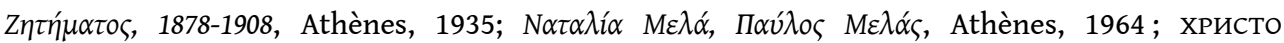
СИЛЯНОВ, ОСВОБОДИТЕЛНИТЕ БОРБИ НА МАКЕДОНИЯ, 2 vols, Sofia, 1933-1943; КОНСТАНТИН ПАНДЕВ, НАЦИОНАЛНО-ОСВОБОДИТЕЛНОТО ДВИЖЕНИЕ В МАКЕДОНИЯ И ОДРИНСКО, 1878-1903, Sofia, 1979 ; СВЕТЛОЗАР ЕЛДЪРОВ, СРЪБСКАТА ВЪОРЪЖЕНА ПРОПАГАНДА В МАКЕДОНИЯ (1901-1912), Sofia, 1993 ; АНГЕЛ ДИНЕВ, ИЛИНДЕНСКАТА ЕПОПЕјА, 2 vols, Skopje, 1987.

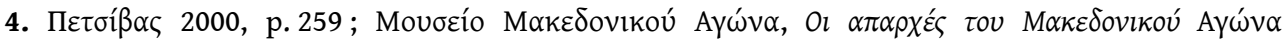
1903-1904, Salonique 1996, p. 228.

5. John Foster Fraser, Pictures from the Balkans, $2^{\text {nd }}$. ed., Londres 1912, pp. 1, 3 et 11.

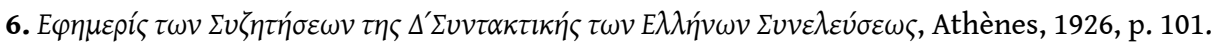

7. Cf. par exemple : IAYE 1907/53, Rapport général du consul grec de Serrès, Antonios Sahtouris

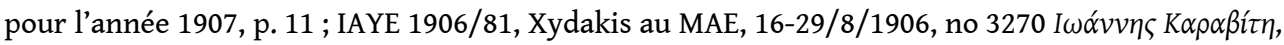

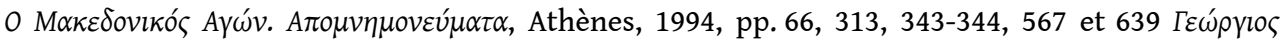

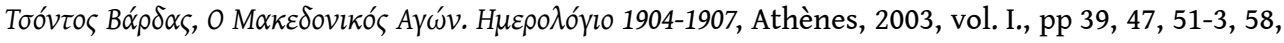

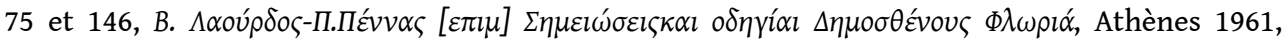
pp. 14-15 et 19.

8. ЦОЧО БИЛЯРСКИ - ИВА БУРИЛКОВА (СЪСТ.), ВМОРО (1893-1919 Г.). ДОКУМЕНТИ НА ЦЕНТРАЛНИТЕ РЪКОВОДНИ ОРГАНИ, Sofia, 2007, p. 179.

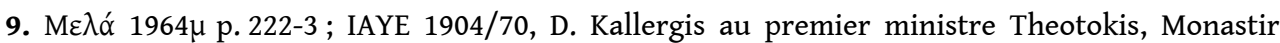
16.7.1904, no 685 ; Mercia MacDermott, For freedom and perfection. The life of Yane Sandansky, Londres, 1988, pp. 168-9, 191-3 \& 444.

10. Charles Eliot, Turkey in Europe, Londres, 1908, p. 271 ; H.N. Brailsford, Macedonia. Its races and future, Londres, 1906, pp. 72, 92, 106, 160, 198, 212 et 314 ; Edith Durham, The burden of the Balkans, Londres, 1905, p.viii, 68, 77, 85, 121-2, 166, 169, 175-6, 179, 203 et 212 ; Fraser 1912, pp. 15 et 176 ; АТАНАС ШОПОВ, ИЗ ЖИВОТА И ПОЛОЖЕНИЕТО НА БЪЛГАРИТЕ В ВИЛАЕТИТЕ, Plovdiv, 1893, p. 68 et 70 ; Jovan Cvijic, Questions balkaniques, Paris-Neuchatel, 1917, p. 74 et 78-79 ; ГЕОРГИ БАЖДАРОВ, ГОРНО

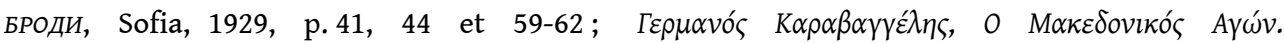

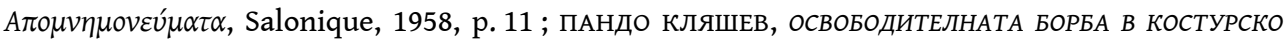
(до 1904 г.), Sofia, 1925, pp. 11 et 111 ; IAYE 1904/70, Stornaris au MAE, Serrès 10.12.1904, no 366 ; IAYE 1906/81, L. Koromilas au MAE, Monastir 8.8.1906, no 1, etc.

11. Pour une description générale de cette dimension politique et sociale de la confrontation nationale, cf. Basil Gounaris, "Social cleavages and national 'awakening' in Ottoman Macedonia",

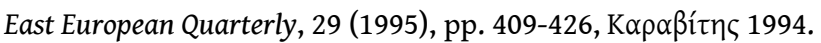

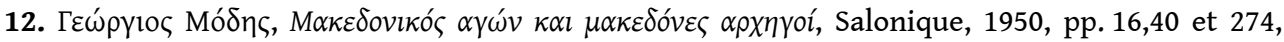

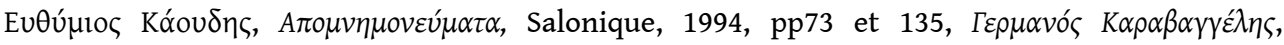

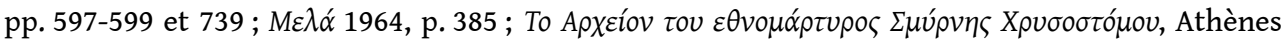
2000, vol. I, pp. 48-49 ; IAYE 1904/74, L. Koromilas au MAE, Salonique, le 28/10/04, no 15.

13. Pallis, Alexandros (1925a), "Racial migrations in the Balkans during the years 1912-1924", The Geographical Journal, 66/4 (du 10/1925), pp. 315-31; Jacques Ancel, La Macédoine. Son évolution contemporaine, Paris, 1930 ; André Wurfbain, L'échange gréco-bulgare des minorités ethniques, Paris, 1930 ; Stephen Ladas, The exchange of minorities, N. York 1932 ; Dimitri Pentzopoulos, The Balkan exchange of minorities and its impact on Greece, Paris - The Hague 1962.

14. Pour une liste des estimations secrètes des divers services administratifs, militaires ou policiers de l'époque : Tasos Kostopoulos, “Counting the 'Other' : Official Census and Classified 
Statistics in Greece (1830-2001)”, Jahrbücher für Geschichte und Kultur Südosteuropas, 5 (2003), pp. 73-74.

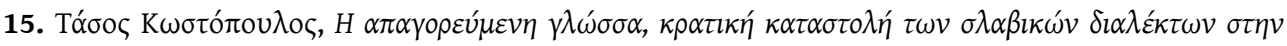
$\varepsilon \lambda \lambda \eta v \imath \kappa \eta ́$ M $\alpha \kappa \varepsilon \delta o v i ́ \alpha$, Athènes, 2000, p. 73-80.

16. АПК, Gouverneur général de la Macédoine Occidentale I. Iliakis au Ministère des Affaires

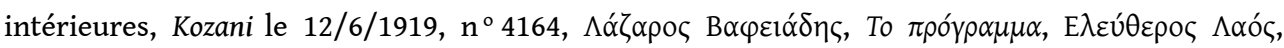
(Edessa) du 6/12/1931.

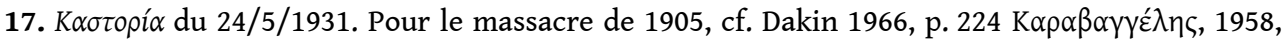

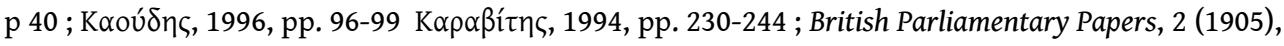

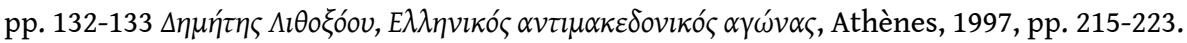

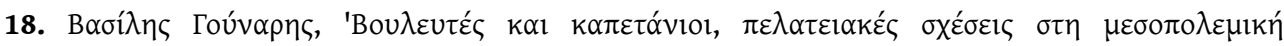

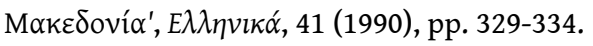

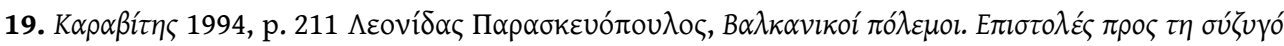

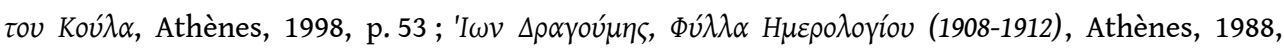
pp. 129-130.

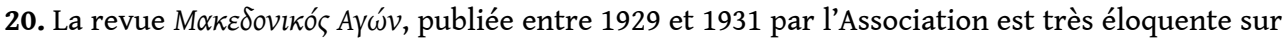
ce point. En fait, « Pavlos Melas » a coopéré étroitement avec l'organisation ouvertement fasciste

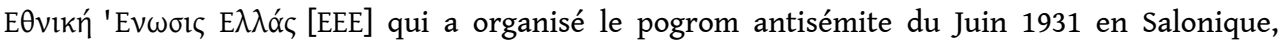

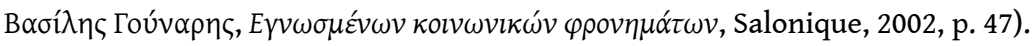

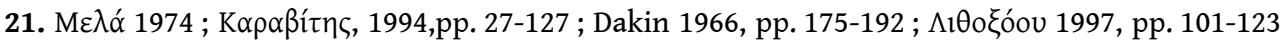

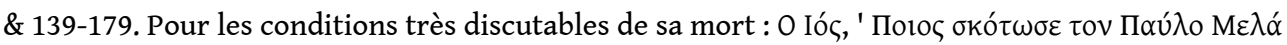

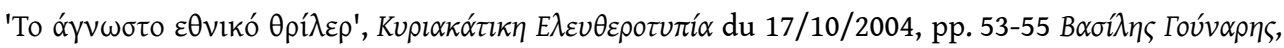

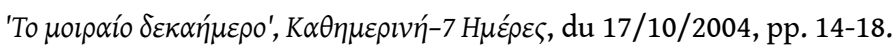

22. Cf. par exemple les observations d'un maitre d'école de l'époque dans le journal local $E \delta \varepsilon \sigma \sigma \alpha$

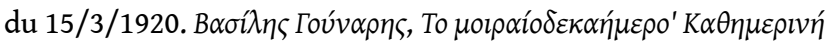

23. À voir surtout le préface de ses Mémoires personnels de "la lutte» : "Peut-être, ces pages peuvent avoir aussi un but pratique. Montrer comment la Macédoine se transformera en ménagerie si jamais ladite

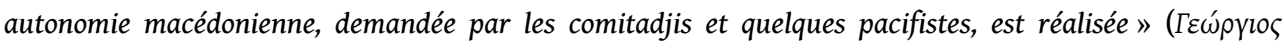

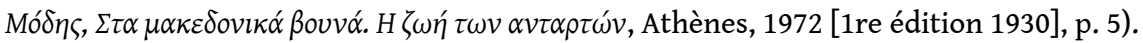

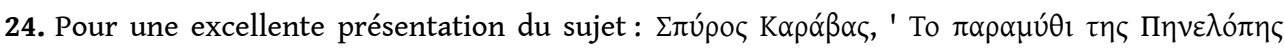

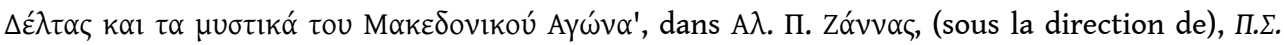

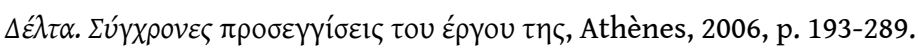

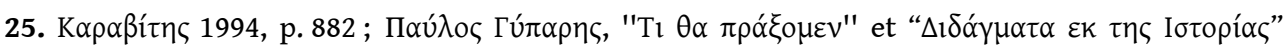

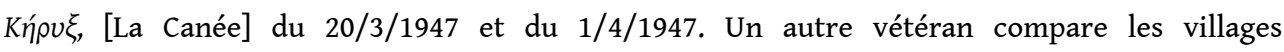

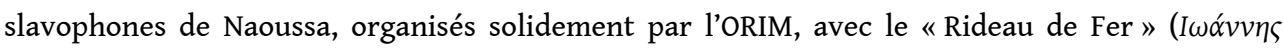

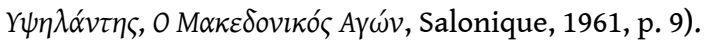

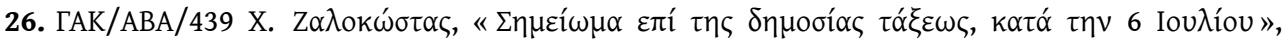
Athènes le 6/7/1946. Pour une présentation et analyse de ce document récemment découvert:

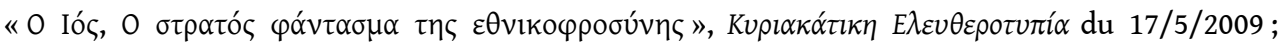
entier, il est accessible sur http://www.iospress.gr/ios2009/ios20090517a.htm.

27. Pour la falsification des sources publiées par IMXA, à voir la critique détaillée de $\Sigma \pi u ́ p o \varsigma$

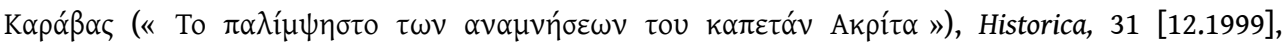

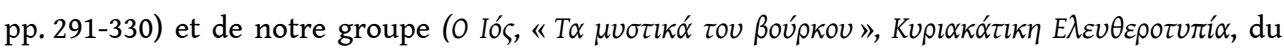
7/7/2002, pp.45-47). Pour les suggestions du président de l'IMXA au Service Historique de l'Armée sur le traitement nécessaire des sources pour " éviter les malentendus » : $\mathrm{A} \Delta \mathrm{I} \Sigma / 92 / 16^{\mathrm{A}}$ / 89-91, Stilpon Kyriakides a DIS, Salonique le 27/5/1959, No 2136.

28. À voir par exemple la revue Aristotelis de Florina, très nationaliste et subventionnée par des « crédits secrets » du Ministère des Affaires extérieures, destinés à l'assimilation linguistique et nationale de la population slavophone. 
29. $\mathrm{A} \Phi \Delta / 129 / 98$, « Projet d'étude de l'histoire moderne de la Macédoine, préparé par l'IMXA » (1962) ; AФ $\Delta / 96 / 208,1^{\text {re }}$ Direction de MAE (K. Heimarios), « Notice sur la rédaction de l'Histoire de la Macédoine ", Athènes le 4/6/1963, Classée «Très Secrète »; ПA, 1re Direction de MAE (D. Bitsios), « Note pour M. le Ministre ", mai 1965, Classée «Très Secrète »; ПА, 1re Direction de MAE , « Notice. Institut des Études balkaniques (IMXA) », Athènes le 26/5/1965, Classée «Très Secrète».

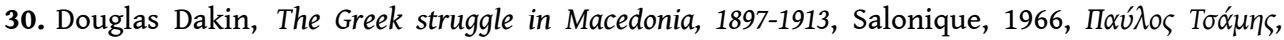

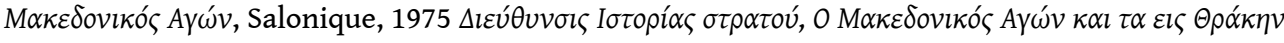

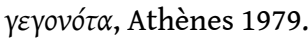

31. L'histoire officielle de l'Armée a été simplement traduite en 1998 de la forme linguistique archaïsante de katharevousa dans la langue démotique utilisée aujourd'hui par tous les Grecs $(\Delta \mathrm{I} \Sigma$,

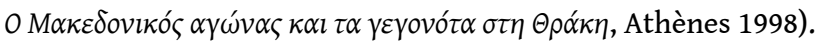

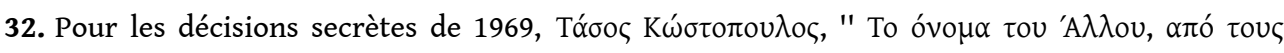

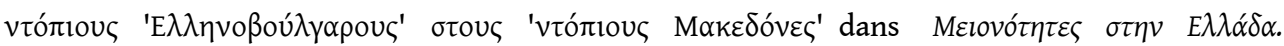

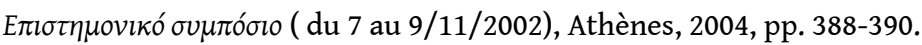

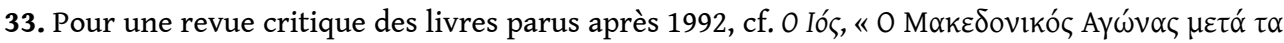

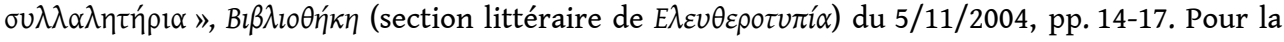
dénonciation, par un apologiste modéré de l'historiographie officielle, de cette "tentative bibliographique - même limitée - qui, pour la première fois dans le territoire grec, vise clairement à faire

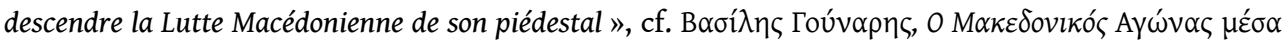

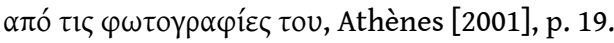

\section{RÉSUMÉS}

L'historiographie grecque a, selon les époques, pour raisons idéologiques, présenté fort différemment les guérillas nationalistes des années 1900 en Macédoine.

La campagne déclenchée par les bandes de guérilla nationalistes entre 1904 et 1908 pour gagner de force «le territoire des âmes » de la population macédonienne, a eu plusieurs aspects ; sa dimension de guerre civile, déchirement fratricide des communautés chrétiennes slavophones, a été reconnue à l'époque par les principaux acteurs et par la plupart des observateurs extérieurs. Son traitement dans l'historiographie grecque est très éclairant sur la fonction idéologique que celle-ci a été appelée à remplir depuis un siècle ; cet article présente les quatre étapes que l'on peut distinguer dans ce traitement depuis un siècle, variations liées évidemment au contexte politique balkanique et intérieur grec : 1910-40, 1941-1974, 1974-1992 et depuis 1992.

The war engaged by the nationalist guerrilla bands between 1904 and 1908 to obtain by force "the souls territory" of the Macedonian population, had different forms. The civil war aspect, bloody shooting between brothers of Christian slavophones communities, was recognized at the time by the main actors of it ans the foreign observers. Its treatment by the Greek historiography shows a very clear light on the ideological function it has been supposed to assume since one century and over. This paper presents the four moments in this treatment whose variations are related, of course, to the political Greek and Balkan context: 1910-1940, 1941-1974, 1974-1992 and from 1992. 
INDEX

motsclestr Balkanlar, Yunan İç Savaşı (1946-1949), Yunanistan, Makedonya, Monastir, Bitola motsclesmk БАЛКАНОТ, ГРЦИЈА, МАКЕДОНИЈА, БИТОЛА

Thèmes : Histoire

Mots-clés : guerillas nationalistes, Andartes, Comitadji, historiographie grecque, Delta Pénélope (1874-1941), luttes macédoniennes, Exarchat, Lutte Macédonienne, propagande nationaliste, Pavlos Mélas (1870-1904), Mürzsteg (programme), ORIM/VRMO, Tcheknik, Armée démocratique

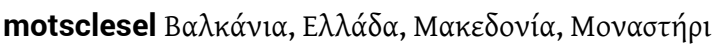

Index chronologique : guerre civile de Macédoine (1895-1908)

Index géographique : Balkans, Grèce, Macédoine, Monastir

Keywords : Macedonian wars (1895-1908), Civil war, Balkans, Greek historiography, Greece, Macedonia, Nationalistic guerilla, Propaganda, History

\section{AUTEUR}

\section{TASOS KOSTOPOULOS}

Historien et journaliste 